\section{§1. Influence of Magnetic Hysteresis on Quench-Voltage Detection in Large Superconducting Magnets}

Takahata, K.

Here we present a technical problem caused by magnetic hysteresis in quench-voltage detection systems for large superconducting magnets. To detect a quench sensitively, it is necessary to use a balanced bridge circuit for canceling out inductive terminal voltages across a magnet. Fig. 1 illustrates a circuit diagram of the quench detection and protection systems for the LHD poloidal coils. Each pair of coils, which are symmetrically arranged with respect to the equatorial plane of the device, is connected to a dc power supply in series. Therefore, the upper and the lower coils generate the same inductive terminal voltage for all operating modes. The bridge circuit can eliminate the inductive voltage using a center tap at the joint between the upper and lower coils. Fig. 2 shows the observed output voltage from the bridge circuit when the IV coils are energized to a maximum current of $15.6 \mathrm{kA}$. Also shown is the energizing current. The graph indicates that two types of out-of-balance voltages appear. One is a mound-shaped change with a peak of approximately $10 \mathrm{mV}$ (indicated by " $\mathrm{A}$ "). The second is a sharp spike with a peak of over $100 \mathrm{mV}$ (indicated by "B"). These spikes occur only after the current has started or stopped changing. The observations suggest that the out-of-balance voltage is caused by a changing magnetization related to superconductivity.

The quench-voltage detection system can balance the voltage due to the magnetization as well as the inductive voltage if the two coils have the same magnetic properties. However, the observations of out-of-balance voltages suggest that there is a difference in magnetization between the two coils. We therefore tried to determine the conditions that reproduce the observed out-of-balance voltages using the analytical methods and assuming different magnetizations. As a result, we were able to confirm differences not only in the penetration field but also in the coupling time constant by our analysis. First, we assumed that the superconducting filaments in the upper coil have a $10 \%$ higher penetration field than those in the lower coil. Fig. 3 shows the experimental and the calculated waveforms. The comparison between these two waveforms confirms that the mound-shaped voltages, indicated by " $\mathrm{A}$ " in Fig. 2, are caused by the difference in penetration field between the coils. In addition, the sharp spikes, indicated by "B" in Fig. 2, can be explained by the difference in coupling time constant.

The observed out-of-balance voltages in the LHD poloidal coils can be explained by the difference in magnetization between the balanced coils. However, this phenomenon is not specific to these coils. For magnets as large as the LHD magnet system, the terminal voltage due to magnetization can influence the quench-voltage detection. To balance the terminal of two coils, it is necessary to select coils that have the same magnetic properties.

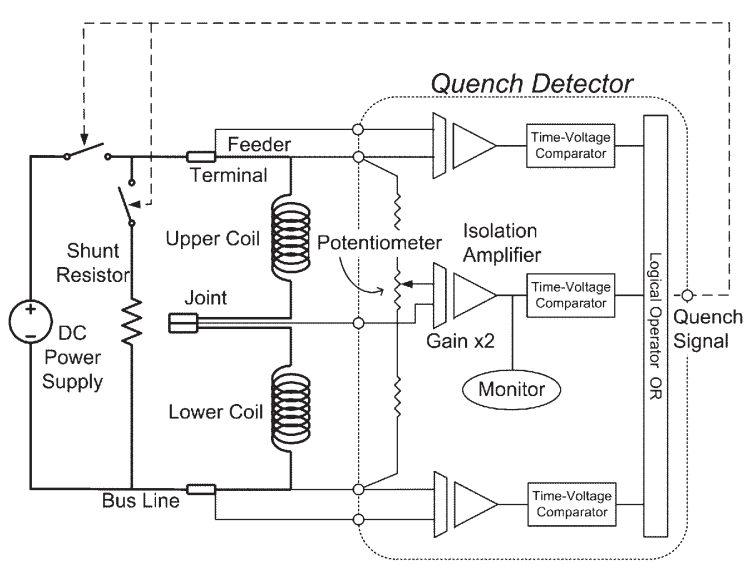

Fig. 1. Circuit diagram of the quench detection and protection systems for the LHD poloidal coils.

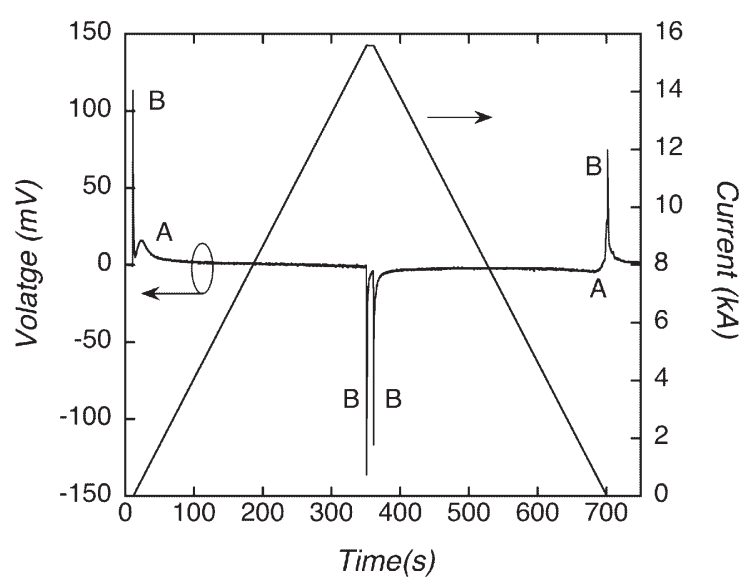

Fig. 2. Observed out-of-balance voltage in the quench detector for the IV coils. The energizing current is also shown

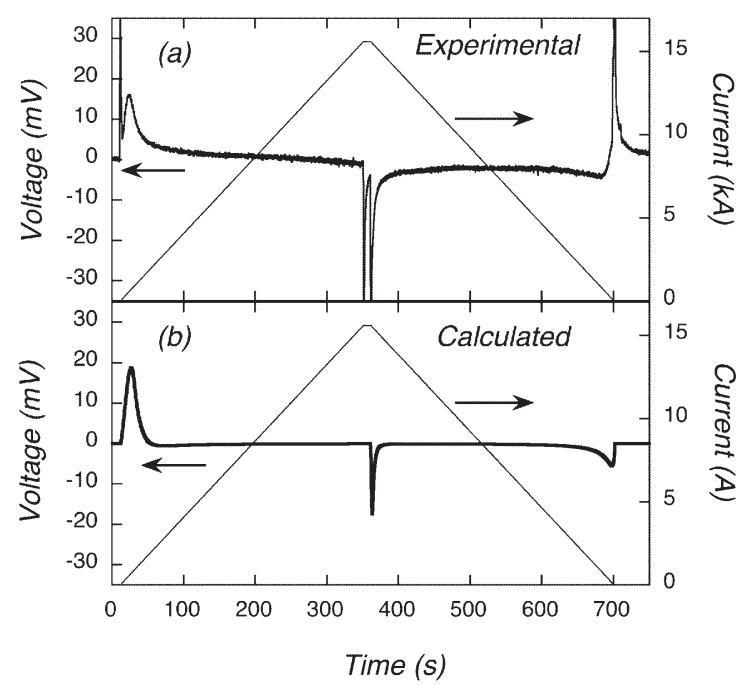

Fig. 3. Comparison of (a) experimental and (b) calculated out-of-balance voltages, assuming a difference in penetration field. 